\title{
ON ONE PROBLEM OF CONTROL OF VOLTAGE DURING SIGNAL TRANSMISSION IN A LONG LINE
}

\author{
N.D. Livanov, nikita.livanov.mail@gmail.com, \\ V.I.Ukhobotov, ukh@csu.ru \\ Chelyabinsk State University, Chelyabinsk, Russian Federation
}

\begin{abstract}
This article discusses the problem of controlling the process of electromagnetic oscillations in a long-distance transmission line. A long line is understood as an electrical line formed, in the simplest case, by two parallel current conductors, the length of which exceeds the wavelength of the transmitted electromagnetic waves, and the distance between the conductors is much less than the wavelength. Such a line is characterized by four distributed parameters, namely the ohmic resistance of the conductor, inductance, electrical capacity and leakage coefficient. A signal generator is connected to the left end of the line, and the right is grounded, but not of high quality. When transmitting a signal, the current and voltage make small oscillation. The control is the voltage at the left end of the long line, and the disturbance voltage at the right end, the values of which are limited. The boundaries of their permissible values are set. The magnitude of the aggregate of external disturbances acting on the conductor is not known exactly, but only its variation limits are given. Aim. The purpose of the control process is that at a given moment in time, the average value of the voltage value is in a given interval. This average is calculated using the specified function. Materials and methods. To solve the problem, the method of optimizing the guaranteed result was applied. Results. A transition was made to a new one-dimensional variable, with the help of which the considered problem was reduced to a control problem of the same type in the presence of interference. This made it possible to find the necessary and sufficient conditions under which it is possible to achieve the set goals with any admissible set of external forces and interference at the right end. A corresponding algorithm for constructing the law of voltage change at the left end of the conductor is proposed. An example is analyzed that clearly shows how management is built that guarantees the achievement of the set goal. Conclusion. If the found necessary and sufficient conditions are fulfilled, then it is always possible to construct such a law of voltage variation at the left end, which will lead to the achievement of the goal for any admissible interference.
\end{abstract}

Keywords: control, telegraph equations, guaranteed result, interference, long-distance transmission line.

\section{Introduction}

In the study of controlled processes of electromagnetic oscillations in long lines with distributed parameters, mathematical problems of control of hyperbolic equations arise [1-7]. There are problems of controlling the signal transmission process when the exact value of external influences is not known, in practice. External disturbances that generate travelling wave in the lines distort the transmitted signal. When studying problems of this kind, you can apply the method of optimizing the guaranteed result [8]. This method is based on the theory of differential games [9].

In this paper, we consider the problem when the control is the limited in magnitude voltage of the signal generator at the left end of the long line. The interference consists of external disturbances and limited voltage at the right end of the conductor. The exact value of the magnitude of the external disturbance acting on the conductor is not known. Its limits of change are known. The purpose of the control process is that at a given moment in time the average value of the voltage value is in a given interval. The average is calculated using the specified function The problem is reduced to a control problem of the same type in the presence of interference by changing the variable. For problems that are considered in the theory of differential games, optimal controls of the players are constructed [10].

\section{Formulation of the problem}

Consider a homogeneous, long line length is equal to $l$ with a given resistance $R$, inductance $L$, electrical capacity $C$, leakage factor $G$ [11]. The signal generator is at the left end of the line, and the right 


\section{Управление в технических системах}

end of the conductor is grounded. When transmitting a signal in a long-distance transmission line, the current in the wires is not the same in different sections of the line. It causes a voltage drop in the active resistance of the wires and creates an alternating magnetic field, which in turn induces selfinduction EMF along the entire line. Therefore, the voltage between the wires also does not remain constant along the line $[12,13]$.

Let's associate a coordinate system with a long line, the $\mathrm{X}$ axis of which is directed along the wire. The densities of the aggregate of external disturbances on current and voltage are given by continuous functions $f_{i}(t, x), i=1,2$, where we assume $x$ - abscissa of a certain cross-section of the conductor when the long line is at rest. We denote $J(t, x)$ change in the current and $V(t, x)$ voltage of the line at the time $t$. The control is limited and is the voltage at the left end of the long line. The system of differential equations describing voltage and current fluctuations takes the form of telegraph equations [14].

$$
\left\{\begin{array}{l}
\frac{\partial J(t, x)}{\partial t}=-\frac{1}{L} \frac{\partial V(t, x)}{\partial x}-\frac{R}{L} J(t, x)+f_{1}(t, x), \\
\frac{\partial V(t, x)}{\partial t}=-\frac{1}{C} \frac{\partial J(t, x)}{\partial x}-\frac{G}{C} V(t, x)+f_{2}(t, x),
\end{array}\right.
$$

where $x \in[0, l], t \in[0, p]$.

This system of equations is considered under the given initial conditions

$J(0, x)=g_{1}(x), V(0, x)=g_{2}(x)$,

where the functions $g_{i}(x), i=1,2$ are continuous on the segment $[0, l]$. By condition, the voltage at the ends of the line is limited. Therefore, they can be written as

$$
\begin{aligned}
& V(t, l)=\lambda_{1}(t)-\alpha_{1}(t) \eta,|\eta| \leq 1, \alpha_{1}(t) \geq 0, \\
& V(t, 0)=\lambda_{2}(t)-\alpha_{2}(t) \xi,|\xi| \leq 1, \alpha_{2}(t) \geq 0 .
\end{aligned}
$$

The parameter $\xi$ is a control, and $\eta$ is a interference.

We assume that the densities $f_{i}(x, t)$ of the magnitudes of external disturbances are not exactly known. The following estimates are known

$$
\overline{\bar{f}}_{l}(t, x) \leq f_{i}(t, x) \leq \bar{f}_{l}(t, x), i=1,2, x \in[0, l], t \in[0, p] .
$$

Where $\bar{f}_{l}(t, x)_{i}:[0, p] \times[0, l] \rightarrow \mathbb{R}$, and $\overline{\bar{f}}_{l}(t, x):[0, p] \times[0, l] \rightarrow \mathbb{R}$ are continuous functions.

Let's set the number $k \in \mathbb{R}, \varepsilon \geq 0$. The purpose of the choice of control $\xi$ (4) is to implement the inequality

$$
\left|\int_{0}^{l}\left(V(p, x) \sigma_{1}(x)+J(p, x) \sigma_{2}(x)\right) d x-k\right| \leq \varepsilon
$$

for any realization of external disturbances, the density of which satisfies the condition (5). Here the functions $\sigma_{i}:[0,1] \rightarrow \mathbb{R}, i=1,2$ are continuous and satisfy the conditions

$$
\sigma_{i}(0)=\sigma_{i}(l)=0 \text {. }
$$

\section{Formalization of the problem}

Let us describe the admissible rule for the formation of control $\xi$ (4). It means that each moment of time $0 \leq \vartheta<p$ and each possible function $V(\vartheta, x), J(\vartheta, x)$ is assigned a function $\xi:[\vartheta, p] \rightarrow[0,1]$. This rule will be denoted

$$
\xi(t)=\mathcal{N}(t, V(\vartheta, \cdot), J(\vartheta, \cdot)), t \in[\vartheta, p] .
$$

We fix the partition of the segment $[0, p]$

$$
\omega: 0<t_{0}<t_{1}<\cdots<t_{j}<t_{j+1}<\cdots<t_{m+1}=p
$$

where a diameter $d(\omega)=\max _{0 \leq j \leq p}\left(t_{j+1}-t_{j}\right)$. Let us fix control (8), the density of external disturbances $f_{i}(t, x)$ at $x \in[0, l], 0 \leq t \leq p$. Let us construct solutions $V_{\omega}(t, x), J_{\omega}(t, x) 0 \leq x \leq l, 0 \leq t \leq p$, to problem (1)-(3).

Assume $g_{i_{0}}(x)=g_{i}(x), i=1,2$ at $0 \leq x \leq l$. The functions $V_{\omega}(x, t)$ и $J_{\omega}(t, x)$ at $t_{0} \leq t \leq t_{1}$, $0 \leq x \leq l$ are defined as a solution to the following problem:

$$
\begin{aligned}
& \left\{\begin{array}{l}
\frac{\partial J_{\omega}\left(t_{j}, x\right)}{\partial t}=-\frac{1}{L} \frac{\partial V_{\omega}\left(t_{j}, x\right)}{\partial x}-\frac{R}{L} J_{\omega}\left(t_{j}, x\right)+f_{1}\left(t_{j}, x\right), \\
\frac{\partial V_{\omega}\left(t_{j}, x\right)}{\partial t}=-\frac{1}{C} \frac{\partial J_{\omega}\left(t_{j}, x\right)}{\partial x}-\frac{G}{C} V_{\omega}\left(t_{j}, x\right)+f_{2}\left(t_{j}, x\right),
\end{array}\right. \\
& J_{\omega}\left(t_{j}, x\right)=g_{1}(x), V_{\omega}\left(t_{j}, x\right)=g_{2}(x), \\
& V_{\omega}(t, 0)=\lambda(t)-\alpha(t) \xi, V_{\omega}(t, l)=0,
\end{aligned}
$$


$\xi_{i}=\mathcal{N}\left(t, V_{\omega}(\vartheta, \cdot), J_{\omega}(\vartheta, \cdot)\right)$.

Where $j=0, x \in[0, l], t \in\left[t_{0}, t_{1}\right]$.

Let the functions $V_{\omega}(x, t), J_{\omega}(t, x)$ be defined for $t_{0} \leq t \leq t_{i-1}, 0 \leq x \leq l$. Suppose $g_{1 j}(x)=$ $=J_{\omega}\left(t_{j-1}, x\right), g_{2 j}(x)=V_{\omega}\left(t_{j-1}, x\right)$. We construct the functions $V_{\omega}(t, x), J_{\omega}(t, x)$ at $t_{j-1} \leq t \leq t_{j}$ using formulas (9)-(12).

We say that control (8) guarantees the fulfillment of the set goal (6) if for any number $w>\varepsilon$ there is a number $\rho>0$ such that for any partition $\omega$ with diameter $d(\omega)<\rho$ and for any continuous functions $f_{i}(t, x)$, satisfying the condition (5), the inequality

$\left|\int_{0}^{l}\left(V_{\omega}(p, x) \sigma_{1}(x)+J_{\omega}(p, x) \sigma_{2}(x)\right) d x-k\right| \leq w$.

\section{Transition to a one-dimensional problem}

Let the functions $\psi(\tau, x), \varphi(\tau, x)$ at $0 \leq x \leq l, 0 \leq \tau \leq p$ be a solution to the following problem:

$\left\{\begin{array}{l}\frac{\partial \varphi(\tau, x)}{\partial \tau}=\frac{1}{L} \frac{\partial \psi(\tau, x)}{\partial x}-\frac{G}{C} \varphi(\tau, x), \\ \frac{\partial \psi(\tau, x)}{\partial \tau}=\frac{1}{C} \frac{\partial \varphi(\tau, x)}{\partial x}-\frac{R}{L} \psi(\tau, x) .\end{array}\right.$

$\varphi(\tau, 0)=0, \varphi(\tau, l)=0,0 \leq \tau \leq p$,

$\varphi(0, x)=\sigma_{1}(x), \psi(0, x)=\sigma_{2}(x), 0 \leq x \leq l$.

It follows from equality (7) that the matching conditions at the ends of the segment are satisfied.

From equalities (5) we obtain that

$$
\begin{aligned}
& \int_{0}^{l} f_{1}(t, x) \psi(p-t, x) d x=\beta_{1}(t)+\gamma_{1}(t) \eta_{1},\left|\eta_{1}\right| \leq 1 \\
& \int_{0}^{l} f_{2}(t, x) \varphi(p-t, x) d x=\beta_{2}(t)+\gamma_{2}(t) \eta_{2},\left|\eta_{2}\right| \leq 1
\end{aligned}
$$

When at $i=1,2$

$$
\begin{gathered}
\beta_{i}(t)=\frac{1}{2} \int_{0}^{1}\left(\overline{\bar{f}}_{l}(t, x)+\bar{f}_{l}(t, x)\right) \psi(p-t, x) d x, \\
\gamma_{i}(t)=\frac{1}{2}\left|\int_{0}^{1}\left(\bar{f}_{l}(t, x)-\overline{\bar{f}}_{l}(t, x)\right) \psi(p-t, x) d x\right| \geq 0 .
\end{gathered}
$$

Suppose

$$
\theta_{\omega}(t)=\int_{0}^{l}\left(J_{\omega}(t, x) \psi(p-t, x)+V_{\omega}(t, x) \varphi(p-t, x)\right) d x .
$$

That

$$
\begin{aligned}
& \dot{\theta}_{\omega}(t)=\int_{0}^{l}\left(\frac{\partial J_{\omega}(t, x)}{\partial t} \psi(p-t, x)+\frac{\partial V_{\omega}(t, x)}{\partial t} \varphi(p-t, x)\right) d x- \\
& -\int_{0}^{l}\left(J_{\omega}(t, x) \frac{\partial \psi(p-t, x)}{\partial \tau}+V_{\omega}(t, x) \frac{\partial \varphi(p-t, x)}{\partial \tau}\right) d x .
\end{aligned}
$$

Let us take into account equations (9), (14) and (17), (18). We get

$$
\begin{aligned}
& \dot{\theta}_{\omega}(t)=\beta_{1}(t)+\gamma_{1}(t) \eta_{1}+\beta_{2}(t)+\gamma_{2}(t) \eta_{2}-\int_{0}^{l}\left(J_{\omega}(t, x) \frac{\partial \psi(p-t, x)}{\partial \tau}+V_{\omega}(t, x) \frac{\partial \varphi(p-t, x)}{\partial \tau}\right) d x+ \\
& +\int_{0}^{l}\left(\left(-\frac{1}{L} \frac{\partial V_{\omega}(t, x)}{\partial x}-a_{2} J_{\omega}(t, x)\right) \psi(p-t, x)+\left(-\frac{1}{c} \frac{\partial J_{\omega}(t, x)}{\partial x}-b_{2} V_{\omega}(t, x)\right) \varphi(p-t, x)\right) d x,
\end{aligned}
$$

at $t_{j} \leq t \leq t_{j+1}$.

Further, integrating by parts and taking into account the boundary conditions (11), (15), we obtain $\int_{0}^{l}\left(\frac{\partial V_{\omega}(t, x)}{\partial x} \psi(p-t, x)\right) d x=\left(\lambda_{1}(t)-\alpha_{1}(t) \eta\right) \psi(p-t, l)-$

$-\left(\lambda_{2}(t)-\alpha_{2}(t) \xi\right) \psi(p-t, 0)-\int_{0}^{l}\left(V_{\omega}(t, x) \frac{\partial \psi(p-t, x)}{\partial x}\right) d x$,

$\int_{0}^{l}\left(\frac{\partial J_{\omega}(t, x)}{\partial x} \varphi(p-t, x)\right) d x=-\int_{0}^{l}\left(J_{\omega}(t, x) \frac{\partial \varphi(p-t, x)}{\partial x}\right) d x$.

From this and (20) it follows that at $t_{j} \leq t \leq t_{j+1}$,

$\beta(t)=\beta_{1}(t)+\beta_{2}(t)+\lambda_{1}(t), \gamma(t)=\gamma_{1}(t)+\gamma_{2}(t)+\alpha_{1}(t) \geq 0$,

$v_{j}=\frac{\gamma_{1}(t)}{\gamma(t)} \eta_{1}+\frac{\gamma_{2}(t)}{\gamma(t)} \eta_{2}+\frac{\alpha_{1}(t)}{\gamma(t)} \eta,\left|v_{j}\right| \leq 1$

$\dot{\theta}_{\omega}(t)=-\left|\frac{1}{L} \alpha_{2}(t) \psi(p-t, 0)\right| u_{j}+\gamma(t) v_{j}+\beta(t)+\frac{1}{L} \lambda_{2}(t) \psi(p-t, 0)$. 


\section{Управление в технических системах}

When

$\xi=\operatorname{sign}\left(\frac{1}{L} \alpha_{2}(t) \psi(p-t, 0)\right) u$.

Suppose, that $\operatorname{sign} 0=1$.

Denote

$z_{\omega}(t)=\theta_{\omega}(t)+\int_{t}^{p}\left(\beta(r)+\frac{1}{L} \lambda_{2}(r) \psi(p-r, 0)\right) d r-k$.

That for $t_{j} \leq t \leq t_{j+1}$,

$\dot{z}_{\omega}(t)=-a(t) u_{j}+b(t) v_{j},\left|u_{i}\right| \leq 1,\left|v_{i}\right| \leq 1$.

Here it is denoted

$a(t)=\left|\frac{1}{L} \alpha_{2}(t) \psi(p-t, 0)\right| \geq 0, b(t)=\gamma(t) \geq 0$.

Further, taking into account condition (11) and formulas (19), (22), we rewrite inequality (13) in the following form:

$\left|z_{\omega}(p)\right| \leq w$.

\section{Termination possibility conditions in a one-dimensional problem}

Consider the one-dimensional problem (23), (25). Note that functions (24) are continuous. Let's build broken lines

$$
z_{\omega}(t)=z_{\omega}\left(t_{j}\right)-\int_{t_{j}}^{t} a(r) d r u_{j}+\int_{t_{j}}^{t} b(r) d r v_{j}, t_{j} \leq t \leq t_{j+1},
$$

where $z_{\omega}(0)=z(0)$ is the initial condition. The family of these broken lines is uniformly bounded and equicontinuous, defined on the segment $[0, p][10, \mathrm{p} .46]$. By Arzela's theorem [15, p. 104] from any sequence of polygonal lines (26), one can select a subsequence that converges uniformly to the segment $[0, p]$.

Let in (26)

$u_{j}=\operatorname{sign} z_{\omega}\left(t_{j}\right), j=\overline{0, m}$,

and the function $z(t)$ at $0 \leq t \leq p$ is the uniform limit of the polygonal sequence $z_{\omega_{n}}(t)$ (26), for which the diameter of the partition $d\left(\omega_{n}\right) \rightarrow 0$. Then [10, Theorem 8.1] the inequality

$$
|z(p)| \leq F(z(0)) \text {. }
$$

Here it is denoted

$$
F(z)=\max \left(|z|+\int_{0}^{p}(b(r)-a(r)) d r ; \max _{0 \leq \tau \leq p} \int_{\tau}^{p}(b(r)-a(r)) d r\right) .
$$

Let the number $\varepsilon \geq F(z(0))$. Then it can be shown that for any number $w>\varepsilon$ there is a number $\delta>0$ such that inequality (25) holds for any broken line (26) with the partition diameter $d(\omega)<\delta$ and control (27).

Let in (26)

$v_{j}=\operatorname{sign} z_{\omega}\left(t_{j}\right), j=\overline{0, m}$,

and the function $z(t)$ at $0 \leq t \leq p$ is the uniform limit of a sequence of polygonal lines $z_{\omega_{n}}(t)$ (26), for which $d\left(\omega_{n}\right) \rightarrow 0$. Then [10, Theorem 8.2], the inequality

$|z(p)| \geq F(z(0))$.

From this it can be obtained that if the numbers $\varepsilon<w<F(z(0))$, then there exists a number $\delta>0$ such that то $\left|z_{\omega}(p)\right|>w$, for any broken line $z_{\omega}(t)$ (26) with the partition diameter $d(\omega)<\delta$ and with $v_{j}(28)$.

Thus, it is possible to construct control (8), which guarantees the fulfillment of the set goal (13) if and only if $F(z(0)) \leq \varepsilon$.

From formulas (21), (27) we obtain that

$\xi=\operatorname{sign}\left(z a_{2}(t) \int_{0}^{l} \psi(p-t, x) d x\right) u$.

When $z$ is defined by formulas (19) and (22) with given in (22) $V_{\omega}(x, t), J_{\omega}(t, x)$ on $V(t, x), J(t, x)$.

\section{Example}

Let the function

$\sigma_{2}(x)=\frac{b^{2}}{\frac{1}{a^{2}}-\left(\frac{\pi}{l}\right)^{2}} \sin \left(\frac{\pi}{l} x\right)$ 
when

$$
a=\frac{1}{\sqrt{L C}}, b=\frac{\sqrt{\frac{1}{4}(R C+G L)^{2}-L R C G}}{L C},
$$

then condition (7) is satisfied. Consider the function

$$
\psi(\tau, x)=b^{2} e^{-\frac{\sqrt{L C}(R C+G L) \tau}{2}}\left(\frac{1}{\frac{1}{a^{2}}-\left(\frac{\pi}{l}\right)^{2}} \sin \left(\frac{\pi}{l} x\right)+\frac{1}{a^{2}-1} \sin (\tau)\right),
$$

that satisfies Eq. (14) and conditions (15), (16). Substitute the function $\psi(x, \tau)$ into formula (19) at $V_{\omega}(x, t)=V(t, x)$. Then it follows from $(22)$ that

$$
\begin{aligned}
& z(t)=b^{2} \int_{0}^{l}\left(V_{\omega}(t, x) e^{-\frac{\sqrt{L C}(R C+G L)(p-t)}{2}}\left(\frac{\sin \left(\frac{\pi}{l} x\right)}{\frac{1}{a^{2}}-\left(\frac{\pi}{l}\right)^{2}}+\frac{\sin (p-t)}{a^{2}-1}\right)\right) d x+ \\
& +\int_{t}^{p}\left(\beta(r)+\frac{a_{1} b^{2}}{a^{2}-1} \lambda(r) e^{-\frac{\sqrt{L C}(R C+G L)(p-r)}{2}} \sin (p-r)\right) d r-k .
\end{aligned}
$$

\section{Conclusion}

The impact of interference on a long line leads to distortion of information, to a decrease in the quality of transmission and subsequent processing of data until the destruction of the communication lines themselves. However, it is possible to construct such a law of voltage variation at the left end, which will lead to the achievement of the goal with any admissible interference, with known estimates of the set of negative effects and the fulfillment of the necessary and sufficient conditions found. The analyzed example clearly demonstrates how the corresponding law of voltage change is constructed.

The research was funded by Russian Foundation for Basic Research and Chelyabinsk Region, project number 20-41-740027.

\section{References}

1. Osipov Yu.S., Oxezin S.P [On the theory of positional control in hyperbolic systems]. Reports of the USSR Academy of Sciences, 1977, vol. 233, no. 4, pp. 551-554. (in Russ.)

2. Lions J.-L. Optimal'noye upravleniye sistemami, opisyvayemymi uravneniyami s chastnymi proizvodnymi [The optimal control of systems described by partial differential equations]. Moscow, Mir Publ., 1972. 416 p.

3. Vasil'yev F.P. Metody resheniya ekstremal'nykh zadach [Methods for solving extreme problems]. Moscow, Nauka Publ., 1981. 400. p.

4. Wu X., Yang X., Shen H., Zhou Q. Research on the Long-distance Transmission. Energy and Power Engineering, 2013, pp. 1293-1297. DOI: 10.4236/epe.2013.54B245

5. Dos Santos M.L., Jardini J.A., Casolari R.P. et al. Power Transmission Over Long Distances: Economic Comparison Between HVDC and Half-Wavelength Line. IEEE Transactions on Power Delivery, 2014, no. 29 (2), pp. 502-509. DOI: 10.1109/TPWRD.2013.2274333

6. Egorov A.I. [Elastic vibration control]. Reports of the Academy of Sciences of the Ukrainian SSR. Ser. Phys.-mat. and tech. sciences, 1986, no. 5, pp. 60-63. (in Russ.).

7. Osipov Yu.S., Korotkiy A.I. [Dynamic modeling of parameters in hyperbolic systems]. Izvestia of the USSR Academy of Sciences. Tech. cybernetics, 1991, no. 2, pp. 154-164. (in Russ.)

8. Krasovskiy N.N. Upravleniye dinamicheskoy sistemoy [Dynamic system control]. Moscow, Nauka Publ., 1985. 520 p.

9. Krasovskiy N.N., Subbotin A.I. Pozitsionnyye differentsial'nyye igry [Positional differential games]. Moscow, Nauka Publ., 1974. 456 p.

10. Ukhobotov V.I. Metod odnomernogo proyektirovaniya v lineynykh differentsial'nykh igrakh $s$ integral'nymi ogranicheniyami [One-dimensional design method in linear differential games with integral constraints. Tutorial]. Chelyabinsk, Chelyabinsk State University Publ., 2005. 124 p.

11. Prasad Rao K.P., Srinivasa Varma P. Analysis of Very Long Distance AC Power Transmission Line. Project: Power Systems, 2019.

12. Zeveke G.V., Ionkin P.A., Netushil A.V., Strakhov S.V. Osnovy teorii tsepey [Fundamentals of circuit theory. Textbook for universities]. Moscow, Energiya Publ., 1975, pp. 513-576. 


\title{
Управление в технических системах
}

13. Abhisek Ukil. Theoretical Analysis of Tuned HVAC Line for Low Loss Long Distance Bulk Power Transmission. International Journal of Electrical Power \& Energy Systems, 2015, no. 73 (4). DOI: 10.1016/j.ijepes.2015.05.021

14. Koshlyakov N.S., Gliner E.B., Smirnov M.M. Osnovnyye differentsial'nyye uravneniya matematicheskoy fiziki [Basic differential equations of mathematical physics]. Moscow, Publishing house phys.-mat. literature, $1962.768 \mathrm{p}$.

15. Kolmogorov A.N., Fomin S.V. Elementy teorii funktsiy i funktsional'nogo analiza [Elements of the theory of functions and functional analysis]. Moscow, Nauka Publ., 1972. 496 p.

Received 5 May 2021

\section{ОБ ОДНОЙ ЗАДАЧЕ УПРАВЛЕНИЯ НАПРЯЖЕНИЕМ ПРИ ПЕРЕДАЧЕ СИГНАЛА В ДЛИННОЙ ЛИНИИ}

\author{
Н.Д. Ливанов, В.И. Ухоботов \\ Челябинский государственный университет, г. Челябинск, Россия
}

\begin{abstract}
В данной статье рассматривается задача управления процессом электромагнитных колебаний в длинной линии. Под длинной линией понимается электрическая линия, образованная в простейшем случае двумя параллельными проводниками тока, длина которых превышает длину волны передаваемых электромагнитных колебаний, а расстояние между проводниками значительно меньше длины волны. Подобная линия характеризуется четырьмя распределёнными параметрами, а именно омическим сопротивлением проводника, индуктивностью, электроёмкостью и коэффициентом утечки. К левому концу линии подключен генератор сигнала, а правый заземлен, но некачественно. При передаче сигнала ток и напряжение совершают малые колебания. Управлением является напряжение на левом конце длинной линии, а помехой напряжение на правом конце, величины которых ограничены. Границы их допустимых значений заданы. Величина совокупности внешних возмущений, действующих на проводник, точно не известна, а заданы только её границы изменения. Цель исследования. Цель процесса управления заключается в том, чтобы в заданный момент времени среднее значение величины напряжения находилось в заданном промежутке. Это среднее значение вычисляется с помощью заданной функции. Материалы и методы. Для решения поставленной задачи был применен метод оптимизации гарантированного результата. Результаты. Был осуществлен переход к новой одномерной переменной, с помощью которой рассматриваемая задача была сведена к однотипной задаче управления при наличии помехи. Это позволило найти необходимые и достаточные условия, при выполнении которых можно осуществить поставленные цели при любой допустимой совокупности внешних возмущений и помехе на правом конце. Предложен соответствующий алгоритм построения закона изменения напряжения на левом конце проводника. Разобран пример, который наглядно показывает, как строится управление, гарантирующее достижение поставленной цели. Заключение. Если выполнены найденные необходимые и достаточные условия, то всегда можно построить такой закон изменения напряжения на левом конце, который приведет к достижению цели при любой допустимой помехе.

Ключевые слова: управление, телеграфные уравнения, гарантированный результат, помеха, длинная линия.
\end{abstract}

\section{Лuтература}

1. Осипов, Ю.С. К теории позищионного управления в гиперболических системах / Ю.С. Осипов, С.П. Охезин // Докл. АН СССР. - 1977. - Т. 233, № 4. - С. 551-554.

2. Лионс, Ж.-Л. Оптимальное управление системами, описываемыми уравнениями с частными производными / Ж.Л. Лионс. - М.: Мир, 1972. - 416 с. 

$400 \mathrm{c}$.

3. Васильев, Ф.П. Методы решения экстремальных задач / Ф.П. Васильев. - М.: Наука, 1981. -

4. Research on the Long-distance Transmission / X. Wu, X. Yang, H. Shen, Q. Zhou // Energy and Power Engineering. - 2013. - P. 1293-1297. DOI: 10.4236/epe.2013.54B245

5. Power Transmission Over Long Distances: Economic Comparison Between HVDC and HalfWavelength Line / M.L. dos Santos, J.A. Jardini, R.P. Casolari et al. // IEEE Transactions on Power Delivery. - 2014. - No. 29 (2). - P. 502-509. DOI: 10.1109/TPWRD.2013.2274333

6. Егоров, А.И. Управление упругими колебаниями / А.И. Егоров // ДАН УССР. Сер. физ-мат. и техн. наук. - 1986. - № 5. - C. 60-63.

7. Осипов, Ю.С. Динамическое моделирование параметров в гиперболических системах / Ю.С. Осипов, А.И. Короткий // Изв. АН СССР. Техн. кибернетика. - 1991. - № 2. - С. 154-164.

8. Красовский, Н.Н. Управление динамической системой / Н.Н. Красовский. - М.: Наука, 1985. $-520 \mathrm{c}$.

9. Красовский, Н.Н. Позиционные дифференщиальные игры / Н.Н. Красовский, А.И. Субботин. - М.: Наука, 1974. - 456 с.

10. Ухоботов, В.И. Метод одномерного проектирования в линейных дифференциальных играх с интегральными ограничениями: учеб. пособие / В.И. Ухоботов. - Челябинск: Челябинский государственный университет, 2005. - $124 \mathrm{c}$.

11. Prasad Rao, K.P. Analysis of Very Long Distance AC Power Transmission Line / K.P. Prasad Rao, P. Srinivasa Varma // Project: Power Systems. -2019.

12. Основы теории цепей: учеб. для вузов / Г.В. Зевеке, П.А. Ионкин, А.В. Нетушил, С.В. Страхов. - М.: Энергия, 1975. - С. 513-576.

13. Abhisek Ukil. Theoretical Analysis of Tuned HVAC Line for Low Loss Long Distance Bulk Power Transmission / Abhisek Ukil // International Journal of Electrical Power \& Energy Systems. - 2015. No. 73 (4). DOI: 10.1016/j.ijepes.2015.05.021

14. Кочляков, Н.С. Основные дифференциальные уравнения математической физики / Н.С. Кошляков, Э.Б. Глинер, М.М. Смирнов. - М.: Изд-во физ.-мат. лит., 1962. -768 с.

15. Колмогоров, А.Н. Элементы теории функций и функционального анализа / А.Н. Колмогоров, С.В. Фомин. - М.: Наука, 1972. -496 с.

Ливанов Никита Дмитриевич, аспирант кафедры теории управления и оптимизации, Челябинский государственный университет, г. Челябинск; nikita.livanov.mail@gmail.com.

Ухоботов Виктор Иванович, д-р физ.-мат. наук, профессор, заведующий кафедрой теории управления и оптимизации, Челябинский государственный университет, г. Челябинск; ukh@csu.ru.

Поступила в редакцию 5 мая 20212.

\section{ОБРАЗЕЦ ЦИТИРОВАНИЯ}

Livanov, N.D. On One Problem of Control of Voltage during Signal Transmission in a Long Line / N.D. Livanov, V.I. Ukhobotov // Вестник ЮУрГУ. Серия «Компьютерные технологии, управление, радиоэлектроника». - 2021. - Т. 21, № 3. - C. 59-65. DOI: $10.14529 /$ ctcr 210306

\section{FOR CITATION}

Livanov N.D., Ukhobotov V.I. On One Problem of Control of Voltage during Signal Transmission in a Long Line. Bulletin of the South Ural State University. Ser. Computer Technologies, Automatic Control, Radio Electronics, 2021, vol. 21, no. 3, pp. 59-65. DOI: $10.14529 / \mathrm{ctcr} 210306$ 